\title{
Evaluation of the relationship between weight gain, fat ectopic accumulation in the liver and hepatic expression of miR-122 in mice fed HFD for different periods.
}

\section{Carolina Panzarin*, Laís A. P. Simino, Mariana C. Mancini, Marcela M. Lage, Marina F. Fontana, Marcio A. Torsoni, Marciane Milanski, Adriana S. Torsoni.}

\begin{abstract}
The number of obese people worldwide is directly associated with the increased prevalence of Non-Alcoholic Fat Liver Disease (NAFLD), a disease characterized by triglycerides accumulation in the liver. Hepatic lipid metabolism may be regulated by microRNAs (miRs) expression. miR-122 is the most expressed miR in the liver and, among other functions, it regulates glucose and lipid metabolism. Insulin resistance appears to be a common event of NAFLD and obesity, since it underlies the genesis of both diseases. Therefore, the aim of the present study was to investigate a possible connection between insulin resistance, obesity, NAFLD development and alterations in miR-122 hepatic expression in mice fed with HFD for different periods.
\end{abstract}

Key words: High-fat diet, non-alcoholic fat liver disease, miR-122.

\section{Introduction}

Obesity is a non-communicable disease characterized by excessive body fat. The number of obese in worldwide is directly associated with the increased prevalence of NonAlcoholic Fat Liver Disease (NAFLD), a pathology that is characterized by triglycerides (TAG) accumulation in the liver, and may progress to Non-Alcoholic Steatohepatitis (NASH), fibrosis, cirrhosis and hepatocarcinoma. Hepatic lipid metabolism can be regulated by microRNAs (miRs) expression. Mir-122 is the most abundant miR in the liver and have multiple functions, such as role in the modulation of lipid and glucose metabolism. The present study aims to investigate a possible relationship of cause and effect between NAFLD development and modulation in miR-122 hepatic expression in mice submitted to HFD for different periods.

\section{Results and Discussion}

Male Swiss mice were fed either control diet or HFD. Animals fed HFD were divided in groups, according to the days of the diet exposure: 1, 3, 7, 15, 30 or 56 days. Mice showed a progression in weight, adiposity and fasting glucose according to HFD exposition. Serum cholesterol also increased with increment of HFD exposition.

In this study, serum TAG varied notably, as well as TAG hepatic levels. There was a significant increase in TAG hepatic accumulation and a decrease in serum TAG in HF56 group.

Additionally was also observed a positive correlation between hepatic TAG and fasting glucose, revealing a connection among these variables.

Hepatic miR-122 was slightly but not significantly increased in HF1 compared to control group, but in all other HFD groups, this level decreased significantly. It suggests that there is a deregulation in lipid metabolism that may be driven by miR-122 expression, since this miRNA participates in lipid homeostasis.

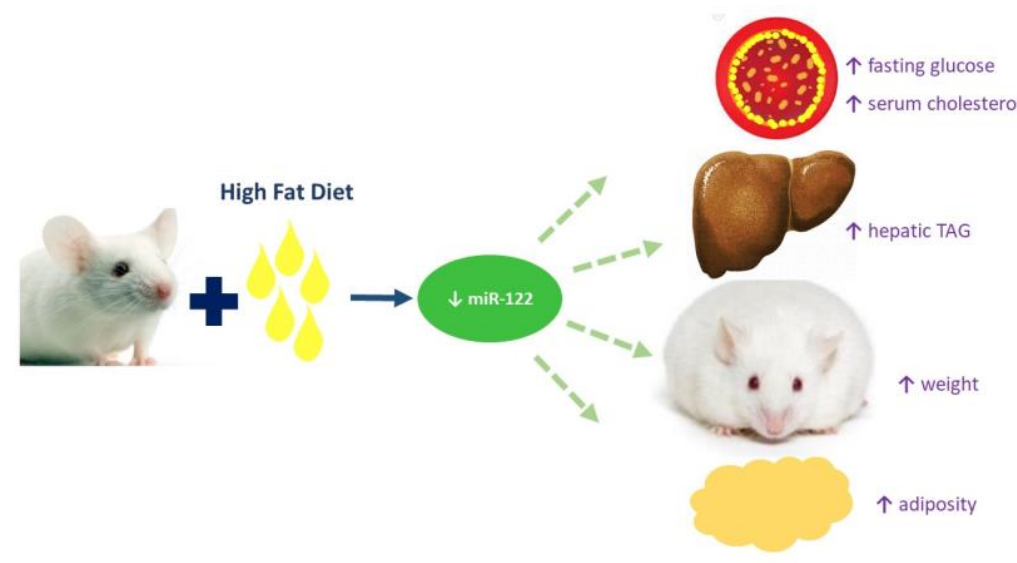

Image 1. Summary of results of the present study.

\section{Conclusions}

High-fat diet consumption lead mice to hepatic miR-122 modulation and metabolic disturbances that precedes changes in body composition. Changes in hepatic miR-122 expression and in its mRNA targets may drive alterations in fat deposition within the liver and hyperglycemia, favoring weight gain and metabolic syndrome development.

\section{Acknowledgement}

I want to thank all members of Laboratório de Distúrbios do Metabolismo (LabDiMe), but specially my adviser Adriana Torsoni and co-adviser Lais Simino. I also thank the financial support from CNPq.

BENATTI, R. O. et al. Maternal high-fat diet consumption modulates hepatic lipid metabolism and microRNA-122 (miR-122) and microRNA-370 (miR370) expression in offspring. Br J Nutr, v. 122, p. 1-11, 2014.

DE PAULA SIMINO, LA. et al. Lipid overload during gestation and lactation can independently alter lipid homeostasis in offspring and promote metabolic impairment after new challenge to high-fat diet. Nutrition \& Metabolism. v. 14, n.16, p. 1-15, 2017.

GIRARD, M. et al. miR-122, a paradigm for the role of microRNAs in the liver. Journal of Hepatology, 2008. 